\section{Relative Salinity Tolerance of Turf-type Saltgrass Selections}

\author{
Y.L. Qian', J.M. Fu, S.J. Wilhelm, D. Christensen, and A.J. Koski \\ Department of Horticulture and Landscape Architecture, Colorado State \\ University, Fort Collins, CO 80523-1173
}

Additional index words. Distichlis spicata var. stricta, halophyte, salinity tolerance, turfgrass

\begin{abstract}
Salt-tolerant turfgrass is highly desirable in areas associated with saline soils or saline irrigation waters. To determine the salt tolerance of 14 saltgrass [Distichlis spicata var. stricta (Greene)] selections, two greenhouse studies were conducted by means of a hydroponic culture system. Five salinity levels (from 2 to $48 \mathrm{dS} \cdot \mathrm{m}^{-1}$ ) were created with ocean salts. In general, turf quality decreased and leaf firing increased as salinity increased. However, varying levels of salt tolerance were observed among selections based on leaf firing, turf quality, root growth, and clipping yield. Selections COAZ-01, COAZ-18, CO-01, and COAZ-19 exhibited the best turf quality and the least leaf firing at 36 and $48 \mathrm{dS} \cdot \mathrm{m}^{-1}$ salinity levels in both Experiments 1 and 2. At the highest salinity level (48 dS' $\left.\mathrm{m}^{-1}\right), \mathrm{COAZ}-18$ and COAZ-19 exhibited the highest root activity among all accessions. Salinity levels that caused $25 \%$ clipping reduction ranged from 21.2 to $29.9 \mathrm{dS} \cdot \mathrm{m}^{-1}$ and were not significantly different among entries. The data on $25 \%$ clipping reduction salinity of saltgrass generated in this study rank saltgrass as one of the most salt-tolerant species that can be used as turf.
\end{abstract}

The use of lesser-quality waters for landscape irrigation in the water-deprived western United States, seawater intrusion into turf facilities located on coastal sites, water conservation, and road deicing have contributed to the increasing salinity problems in many urbanized areas of the western United States (Carrow and Duncan, 1998; Qian and Mecham, 2005). Turfgrass managers are struggling to produce quality turf on sites with high salinity and irrigated with marginal-quality water. With increasing salinity problems, there is a great need to develop and to use salinity-tolerant turfgrasses.

Inland saltgrass [Distichlis spicata var. stricta (L.) Greene], native to Western North America, is a dioecious, rhizomatous, perennial, salt-tolerant, warm-season grass. It is commonly found in saline environments, including saline/alkali salt flats, where it is often a dominant species (Gould, 1968; Hansen et al., 1976; Nielson, 1956). Saltgrass grows in a wide range of soil types, $\mathrm{pH}$ levels, and salinity levels, which makes it one of the most widespread and common halophyte species in the United States (Ungar, 1974). Attributes of saltgrass also include excellent wear, compaction, and drought tolerance (Kopec and Marcum, 2001). Genotypes vary from upright and tall types to compacted and dwarf types, and from cold-hardy to coldtender types (Shahba et al., 2003a, 2003b). These attributes make saltgrass a great candidate for development and use as a turfgrass.

Received for publication 27 June 2006. Accepted for publication 6 Oct. 2006.

The research was supported by the United States Golf Association and the Colorado Agricultural Experimental Station (Projects 658 and 780).

${ }^{1}$ To whom correspondence should be addressed; e-mail Yaling.Qian@colostate.edu.
Work is in progress at Colorado State University and University of Arizona to develop seed- and vegetatively-propagated turf-type saltgrass varieties for targeted use in regions where soil and water salinity are high.

Although saltgrass has been classified as a halophyte (O'Leary and Glenn, 1994), saltgrass genotypes differ in their salinity tolerance. Marcum et al. (2005) evaluated the relative salt tolerance of 21 desert saltgrass accessions and found a great range of salinity tolerance among the saltgrass accessions, but all were more resistant than 'Midiron' bermudagrass [Cynodon dactylon (L.) Pers. var. dactylon $\times C$. transvaalensis Burtt-Davy]. Aschenbach (2006) found that significant differences in salt tolerance existed among different saltgrass source populations. The author suggested that differences in salinity tolerance among saltgrass accessions should be considered when using plant material for landscape restorations.

A series of turf-type saltgrass lines have been selected as parental breeding materials. No information is available about the relative salinity tolerance of these elite, turf-type saltgrass selections. The objective of this study was to screen salinity tolerance and determine the level of salinity tolerance of these advanced saltgrass selections.

\section{Methods and Materials}

Expt. 1. Rhizomes of 14 elite saltgrass lines, CO-01, COAZ-01, COAZ-02, COAZ-06, COAZ-08, COAZ-11, COAZ15, COAZ-16, COAZ-17, COAZ-18, COAZ-19, COAZ-20, COAZ-21, and COAZ-22, were planted in a solution culture system. Individual lines were planted in eight 7-cm-diam. $\times$ 5-cm-deep cups filled with coarse, acid-washed silica sand in Nov.
2002. The cup bottom was removed and covered with nylon screen to hold sand and allow roots to grow through. The cups were placed into holes of a 1.25 -cm-thick plywood lid suspended over a tank containing $30-\mathrm{L}$ of constantly aerated full-strength Hoagland solution. The nylon screen bottom of each cup was submersed $3.0 \mathrm{~cm}$ into the solution. A total of eight tanks were used with each accommodating 14 cups, representing each of the 14 lines. Plants were grown in the greenhouse with the daytime temperature ranging from 23.0 to $29.5^{\circ} \mathrm{C}$ and nighttime temperature ranging from 20.0 to $24.0^{\circ} \mathrm{C}$. To ensure complete establishment, plants were grown for 6 months before initiation of salinity treatment.

These 14 saltgrass selections were successfully established after 6 months culture in the hydroponic system and were further subjected to salinity treatment. Before the initiation of treatment, roots were clipped off and shoots were clipped to $5.0 \mathrm{~cm}$. Four tanks were then subjected to salinity treatment while the other four tanks were maintained as the control (EC of nutrient solution = $2.0 \mathrm{dS} \cdot \mathrm{m}^{-1}$ ). For salinity treatment, instant ocean salt (Aquarium Systems, Mentor, Ohio) was gradually added to increase salinity by $2 \mathrm{dS} \cdot \mathrm{m}^{-1}$ daily until $12 \mathrm{dS} \cdot \mathrm{m}^{-1}$ was reached. The four salinity treatment tanks were held at $12 \mathrm{dS} \cdot \mathrm{m}^{-1}$ for a period of 1 month. During the 1-month period, clippings were harvested every $5 \mathrm{~d}$. At the end of the 1-month period, data were collected on leaf firing, turf quality, root mass, and root viability for both the $12 \mathrm{dS} \cdot \mathrm{m}^{-1}$ salinity treatment and the control. Following data collection, salinity ramping was resumed for salinity treatment until $24 \mathrm{dS} \cdot \mathrm{m}^{-1}$ solution salinity was reached, whereas nutrient solution of the control tanks was maintained at $2.0 \mathrm{dS} \cdot \mathrm{m}^{-1}$. Salinity was again held at these levels for 1 month, and data were collected. The cycle was repeated until the salinity of the growth solution reached 36 and $48 \mathrm{dS} \cdot \mathrm{m}^{-1}$. Selections were maintained at these individual salinity levels for a period of 1 month, respectively, for data collection. At the end of each month's salinity cycle, all roots were harvested at the base of each cup, so that the plants had to regrow their roots as salinity level was being increased. Roots were harvested in the same manner for the control tanks.

For a month at each of $12,24,36$, and $48 \mathrm{dS} \cdot \mathrm{m}^{-1}$ salinity, clippings were harvested every $5 \mathrm{~d}$. At the end of each 1-month salinity exposure, data were collected on leaf firing, turf quality, root mass, and root viability. Turf quality was rated visually on a scale of 0 (brown, dead turf) to 9 (optimum color, density, and uniformity). Turf receiving a rating of 6 was deemed to have acceptable quality. Leaf firing percentage, an indication of salt injury, was determined by visually estimating the total percentage of bleached leaf area. Root viability was quantified by measuring root dehydrogenase activity using triphenyltetrazolium chloride reduction technique (Joslin and Henderson, 1984). About $0.2 \mathrm{~g}$ fresh roots were taken, washed, and 
placed into a test tube filled with $10 \mathrm{~mL}$ of $0.6 \%(\mathrm{w} / \mathrm{v}) 2,3,5$-triphenyltetrazolium chloride (TTC) in $0.05 \mathrm{M} \mathrm{KH}_{2} \mathrm{PO}_{4}$ buffer ( $\mathrm{pH}$ $7.4)+0.05 \%(\mathrm{v} / \mathrm{v})$ wetting agent (Tween-20) . After the samples had been incubated for $20 \mathrm{~h}$ at $26^{\circ} \mathrm{C}$, the TTC solution was poured off and samples were rinsed for 3-4 s with distilled water. Then $10 \mathrm{~mL}$ of $95 \%$ alcohol was added, and samples were kept at room temperature until roots became white $(\approx 24 \mathrm{~h})$. Absorbency of the extract was measured spectrophotometrically at $480 \mathrm{~mm}$. Roots were dried for $48 \mathrm{~h}$ at $75{ }^{\circ} \mathrm{C}$ and weighed. Root activity was expressed as absorbency per gram dry root. At the end of each salinity exposure, roots were harvested and root dry weight was determined.

In Expt. 1, salinity treatment was ramped up, whereas the control was maintained the same throughout the experiment. To assess differences among saltgrass lines, data collected from the control over time were considered as a covariate in analyzing covariance effects according to the general linear models of the Statistical Analysis System (SAS Institute, 2006). Turf quality, leaf firing, clipping yield, root dry weight, and root activity of salinity treatment were then adjusted as if the control over the different time periods for each lines had the same value. Differences among saltgrass selections within each salinity level were separated by the least-significant difference at the 0.05 level of probability.

Expt. 2. Twelve elite experimental lines, including CO-01, COAZ-01, COAZ-02, COAZ-11, COAZ-16, COAZ-17, COAZ-18, COAZ-19, COAZ-20, COAZ-22, COAZ-23, and COAZ-24, were selected for this experiment. Sod pieces of each grass were planted into sixteen 10 -cm-diam. $\times 5$-cm-deep plastic cups filled with a 2-cm layer of Isolite (Sundine Enterprise, Arvada, Colo.). Isolite was chosen in this experiment because of its superior water-holding capacity and low cation-exchange capacity (Qian et al., 2001). Twelve cups consisting of 12 entries were placed into holes of a 1.25 -cm-thick plywood lid, with the lid suspended over a 38-L tank. A total of 16 tanks were used, with each accommodating 12 experimental lines. The tanks contained $36 \mathrm{~L}$ of constantly aerified full strength Hoagland solution, which was replaced weekly. About 20 weeks after planting, just before initiation of salt treatments, roots were clipped at the base of the cups and shoots were clipped to a 2.5-cm height.

The total of 16 tanks were subjected to four salinity treatments. Salinity treatments were applied by adding instant ocean salt gradually during a 10 -d period to obtain EC (EC) values of 6.0, 20.0, 34.0, and $48.0 \mathrm{dS} \cdot \mathrm{m}^{-1}$, respectively, measured by an EC meter (Model CO150, Hach Company, Loveland, Colo.). Because saltgrass is a halophyte, an EC of $6.0 \mathrm{dS} \cdot \mathrm{m}^{-1}$ was considered as the control.

The experimental design was a split-plot with four replications, with salinity level (tank) as the main effect and entries within each tank being the subplot effect. Grasses were exposed to final salinity treatments for 12 weeks. During this period, solution EC of all treatment tanks was measured every $2-3 \mathrm{~d}$ and adjusted when necessary. Data were collected on leaf firing \% (as an indication of salt injury), turf quality, clipping yield, and root mass as described in Expt. 1.

Leaf firing \% was determined weekly, beginning at 5 weeks after initiation of salinity treatments. Means of leaf firing over time are presented for each salinity level. Visual turf quality was rated at the end of the experiment. Throughout the experiment, grasses were clipped weekly to a height of $2.5 \mathrm{~cm}$. Clippings were harvested at 2, 4, and 10 weeks after the initiation of salinity treatments, and dried at $70{ }^{\circ} \mathrm{C}$ for $48 \mathrm{~h}$ for dry weight determination. At the end of the experiment, roots were harvested and dried in a force-draft oven at $70{ }^{\circ} \mathrm{C}$ for $48 \mathrm{~h}$ to determine dry weight.

Salinity and saltgrass selection effects were determined by analysis of variance (SAS Institute, 2006). Selection means at each salinity were separated using Fisher's protected LSD. Regression analysis was performed to define linear or quadratic relation-

Table 1 . Visual turf quality (scale of 0 to 9 , with $9=$ "best") of 14 saltgrass selections under different salinity levels in Expt. 1.

\begin{tabular}{lllll}
\hline & \multicolumn{4}{c}{ Salinity level $\left(\mathrm{dS} \cdot \mathrm{m}^{-1}\right)^{\mathrm{z}}$} \\
\cline { 2 - 5 } Selections & \multicolumn{1}{c}{12} & \multicolumn{1}{c}{24} & 36 & 48 \\
\hline CO-01 & $5.7 \mathrm{bc}^{\mathrm{y}}$ & $6.1 \mathrm{abc}$ & $5.5 \mathrm{ab}$ & $3.7 \mathrm{~b}$ \\
COAZ-19 & $5.3 \mathrm{bcd}_{\text {COAZ-15 }}$ & $5.7 \mathrm{bc}$ & $3.5 \mathrm{~cd}$ & $2.0 \mathrm{cde}$ \\
COAZ-16 & $3.3 \mathrm{e}$ & $2.9 \mathrm{f}$ & $1.0 \mathrm{f}$ & $0.7 \mathrm{e}$ \\
COAZ-20 & $4.3 \mathrm{cde}$ & $4.7 \mathrm{cdef}$ & $4.2 \mathrm{bc}$ & $2.7 \mathrm{bc}$ \\
COAZ-17 & $5.7 \mathrm{bc}$ & $5.0 \mathrm{cde}$ & $2.8 \mathrm{cdef}$ & $2.0 \mathrm{cde}$ \\
COAZ-06 & $7.7 \mathrm{a}$ & $7.0 \mathrm{ab}$ & $3.3 \mathrm{cde}$ & $1.0 \mathrm{e}$ \\
COAZ-08 & $3.5 \mathrm{de}$ & $3.2 \mathrm{ef}$ & $1.5 \mathrm{ef}$ & $2.0 \mathrm{cde}$ \\
COAZ-11 & $5.0 \mathrm{bcde}$ & $5.3 \mathrm{bcd}$ & $3.2 \mathrm{cde}$ & $1.0 \mathrm{de}$ \\
COAZ-21 & $5.0 \mathrm{bcde}$ & $3.7 \mathrm{def}$ & $2.0 \mathrm{def}$ & $5.7 \mathrm{e}$ \\
COAZ-18 & $5.3 \mathrm{bcd}$ & $4.6 \mathrm{cdef}$ & $2.2 \mathrm{def}$ & $5.7 \mathrm{a}$ \\
COAZ-01 & $6.0 \mathrm{abc}$ & $7.0 \mathrm{ab}$ & $6.7 \mathrm{a}$ & $2.7 \mathrm{bc}$ \\
COAZ-22 & $7.7 \mathrm{a}$ & $7.7 \mathrm{a}$ & $7.0 \mathrm{a}$ & $1.3 \mathrm{cde}$ \\
COAZ-02 & $6.0 \mathrm{abc}$ & $6.0 \mathrm{abc}$ & $3.6 \mathrm{~cd}$ & 1.62 \\
LSD & $6.7 \mathrm{ab}$ & $5.7 \mathrm{bc}$ & $2.8 \mathrm{cdef}$ & 1.93 \\
\hline
\end{tabular}

${ }^{\text {zThe }} 2 \mathrm{dS} \cdot \mathrm{m}^{-1}$ level was used as the covariate in statistical analysis, so it is not shown in the table. ${ }^{y}$ Means in the same column followed by the same letter are not significantly different at $P=0.05$.

Table 2. Percent leaf firing (shown as percentage, relative to control) of saltgrass selections exposed to different salinity levels in Expt. 1.

\begin{tabular}{lcccc}
\hline & \multicolumn{4}{c}{ Salinity level $\left(\mathrm{dS} \cdot \mathrm{m}^{-1}\right)^{\mathrm{z}}$} \\
\cline { 2 - 5 } Selections & 12 & 24 & 36 & 48 \\
\hline CO-01 & $8.33 \mathrm{bcde}^{\mathrm{y}}$ & $5.00 \mathrm{bc}$ & $8.33 \mathrm{ab}$ & $11.67 \mathrm{~d}$ \\
COAZ-19 & $5.00 \mathrm{cde}$ & $6.67 \mathrm{bc}$ & $30.00 \mathrm{ab}$ & $50.00 \mathrm{abc}$ \\
COAZ-15 & $5.00 \mathrm{cde}$ & $16.67 \mathrm{ab}$ & $23.33 \mathrm{ab}$ & $51.67 \mathrm{abc}$ \\
COAZ-16 & $15.00 \mathrm{ab}$ & $10.00 \mathrm{bc}$ & $16.67 \mathrm{ab}$ & $33.33 \mathrm{abcd}$ \\
COAZ-20 & $15.00 \mathrm{ab}$ & $31.67 \mathrm{a}$ & $35.00 \mathrm{a}$ & $54.33 \mathrm{ab}$ \\
COAZ-17 & $5.00 \mathrm{cde}$ & $10.00 \mathrm{bc}$ & $13.33 \mathrm{ab}$ & $36.67 \mathrm{abcd}$ \\
COAZ-06 & $0.00 \mathrm{e}$ & $1.67 \mathrm{bc}$ & $6.67 \mathrm{ab}$ & $26.67 \mathrm{bcd}$ \\
COAZ-08 & $11.67 \mathrm{abc}$ & $10.00 \mathrm{bc}$ & $13.33 \mathrm{ab}$ & $13.33 \mathrm{~d}$ \\
COAZ-11 & $30.00 \mathrm{a}$ & $30.00 \mathrm{ab}$ & $63.33 \mathrm{a}$ \\
COAZ-21 & $20.33 \mathrm{a}$ & $10.00 \mathrm{bc}$ & $26.67 \mathrm{ab}$ & $61.00 \mathrm{a}$ \\
COAZ-18 & $10.00 \mathrm{bcd}$ & $6.67 \mathrm{bc}$ & $8.33 \mathrm{ab}$ & $11.67 \mathrm{~d}$ \\
COAZ-01 & $6.00 \mathrm{bcde}$ & $3.33 \mathrm{bc}$ & $5.00 \mathrm{~b}$ & $8.33 \mathrm{~d}$ \\
COAZ-22 & $1.00 \mathrm{de}$ & $0.00 \mathrm{e}$ & $13.33 \mathrm{ab}$ & $13.33 \mathrm{~d}$ \\
COAZ-02 & $5.00 \mathrm{cde}$ & $10.00 \mathrm{bc}$ & $16.67 \mathrm{ab}$ & $11.67 \mathrm{~d}$ \\
LSD & $0.00 \mathrm{e}$ & 15.53 & 29.86 & 30.58 \\
\hline
\end{tabular}

${ }^{\mathrm{z}}$ The $2 \mathrm{dS} \cdot \mathrm{m}^{-1}$ level was used as the covariate in statistical analysis, so it is not shown in the table.

${ }^{\mathrm{y}}$ Means in the same column followed by the same letter are not significantly different at $P=0.05$. 
and COAZ-21. When salinity increased to $36 \mathrm{dS} \cdot \mathrm{m}^{-1}$, only COAZ-01 and COAZ-18 exhibited acceptable quality, and at 48 $\mathrm{dS} \cdot \mathrm{m}^{-1}$, quality of all selections declined to unacceptable. However, at the highest salinity, selections COAZ-01 and COAZ-18 exhibited greater turf quality than other lines, followed by CO-01, COAZ-22, COAZ-16, and COAZ-19 (Table 1).

Relative leaf firing ranges were $0-20 \%$, $0-32 \%, 5-35 \%$, and $8-63 \%$ after 1 month at $12,24,36$, and $48 \mathrm{dS} \cdot \mathrm{m}^{-1}$ salinity growth solutions, respectively (Table 2 ). Selections COAZ-01, COAZ-18, COAZ-02, COAZ-22, COAZ-08, and CO-01 showed less leaf firing than did other lines at $48 \mathrm{dS} \cdot \mathrm{m}^{-1}$ salinity (Table 2). Low leaf firing under high salinity is a good indication of a selection's ability to maintain better turf quality under highly saline conditions.

As salinity increased from 2 to 36 $\mathrm{dS} 7 \cdot \mathrm{m}^{-1}$, root growth increased in selections CO-01, COAZ-01, COAZ-18, COAZ-22, and COAZ-06, but these trends were not as clear in other selections (Table 3). At high salinity levels, COAZ-22, COAZ-01, and COAZ-18 exhibited greater root mass than did COAZ-21, COAZ-15, COAZ-17, COAZ06, COAZ-08, COAZ-11, COAZ-16, and COAZ-19.

For most selections, root activity (viability) increased as salinity increased from 2 to $36 \mathrm{dS} \cdot \mathrm{m}^{-1}$ (Table 4 ). As salinity increased further, root viability decreased. COAZ-18 consistently exhibited the highest root activity among all experimental lines. At the highest salinity level $\left(48 \mathrm{dS} \cdot \mathrm{m}^{-1}\right), \mathrm{COAZ}-18$ and COAZ-19 exhibited the highest root activity among all selections.

On the basis of the number of times in the best statistical category for turf quality, leaf firing, root growth, and root viability in Experiment 1, selections COAZ-18 and COAZ-01, and COAZ-22 have superior salt tolerance. These selections exhibited high turf quality, low leaf firing, and greater root growth under high-salinity conditions. In addition, selection CO-01 also had high turf quality and low leaf firing under high-salinity conditions.

Expt. 2. After 12 weeks of exposure to different levels of salinity, all selections except CO-01, COAZ-19, COAZ-18, and COAZ-11 exhibited increased leaf firing percentages with increasing salinity (Table 5). As seen in Expt. 1, COAZ-18, COAZ-19, CO-01, and COAZ-20 exhibited the least leaf firing under the highest salinity treatment ( 48 $\mathrm{dS} \cdot \mathrm{m}^{-1}$ ), indicating their excellent salinity tolerance. In contrast, selections COAZ-23 and COAZ-02 exhibited the highest leaf firing.

Turf quality of all lines declined to unacceptable quality ratings at the end of the experiment (Table 6). In general, turf quality decreased with increasing salinity. However, the quality decline incited by increasing salinity was less severe for selections CO-01, COAZ-19, and COAZ-18. At the highest salinity $\left(48 \mathrm{dS} \cdot \mathrm{m}^{-1}\right)$, selections CO-01, COAZ-19, COAZ-20, COAZ-18, COAZ-11,

Table 3. Effect of salinity levels on root dry weights (in grams per cup) of 14 saltgrass selections in Expt. 1.

\begin{tabular}{lllll}
\hline & \multicolumn{4}{c}{ Salinity level $\left(\mathrm{dS} \cdot \mathrm{m}^{-1}\right)^{\mathrm{z}}$} \\
\cline { 2 - 5 } Selections & \multicolumn{1}{c}{12} & \multicolumn{1}{c}{24} & \multicolumn{1}{c}{36} & 48 \\
\hline CO-01 & $0.0538 \mathrm{ab}^{\mathrm{y}}$ & $0.1141 \mathrm{abc}$ & $0.1242 \mathrm{abc}$ & $0.1293 \mathrm{bcd}$ \\
COAZ-19 & $0.0423 \mathrm{~b}$ & $0.0565 \mathrm{abc}$ & $0.0441 \mathrm{cde}$ & $0.0307 \mathrm{de}$ \\
COAZ-15 & $0.0458 \mathrm{~b}$ & $0.0556 \mathrm{abc}$ & $0.0678 \mathrm{bcde}$ & $0.0245 \mathrm{e}$ \\
COAZ-16 & $0.0387 \mathrm{~b}$ & $0.0501 \mathrm{bc}$ & $0.0285 \mathrm{de}$ & $0.0394 \mathrm{de}$ \\
COAZ-20 & $0.0524 \mathrm{ab}$ & $0.1274 \mathrm{ab}$ & $0.1163 \mathrm{abcd}$ & $0.1069 \mathrm{cde}$ \\
COAZ-17 & $0.0582 \mathrm{ab}$ & $0.0699 \mathrm{abc}$ & $0.0358 \mathrm{de}$ & $0.0287 \mathrm{de}$ \\
COAZ-06 & $0.0674 \mathrm{ab}$ & $0.01051 \mathrm{abc}$ & $0.1539 \mathrm{ab}$ & $0.0787 \mathrm{de}$ \\
COAZ-08 & $0.0713 \mathrm{ab}$ & $0.0684 \mathrm{abc}$ & $0.0479 \mathrm{cde}$ & $0.0643 \mathrm{de}$ \\
COAZ-11 & $0.0413 \mathrm{~b}$ & $0.0511 \mathrm{bc}$ & $0.0274 \mathrm{e}$ & $0.0374 \mathrm{de}$ \\
COAZ-21 & $0.0426 \mathrm{~b}$ & $0.0443 \mathrm{c}$ & $0.0285 \mathrm{de}$ & $0.0199 \mathrm{e}$ \\
COAZ-18 & $0.0651 \mathrm{ab}$ & $0.1338 \mathrm{a}$ & $0.1460 \mathrm{ab}$ & $0.1864 \mathrm{abc}$ \\
COAZ-01 & $0.0537 \mathrm{ab}$ & $0.1015 \mathrm{abc}$ & $0.1530 \mathrm{ab}$ & $0.2340 \mathrm{ab}$ \\
COAZ-22 & $0.0992 \mathrm{a}$ & $0.1085 \mathrm{abc}$ & $0.1729 \mathrm{a}$ & $0.2401 \mathrm{a}$ \\
COAZ-02 & $0.0680 \mathrm{ab}$ & $0.1186 \mathrm{abc}$ & $0.0840 \mathrm{bcde}$ & $0.0903 \mathrm{cde}$ \\
LSD & 0.0492 & 0.0790 & 0.0883 & 0.1045 \\
\hline
\end{tabular}

${ }^{\text {zThe }} 2 \mathrm{dS} \cdot \mathrm{m}^{-1}$ level was used as the covariate in statistical analysis, so it is not shown in the table.

${ }^{\mathrm{y}}$ Means in the same column followed by the same letter are not significantly different at $P=0.05$.

Table 4. Effect of different salinity levels on root viability (absorption per gram of root dry weight) of saltgrass selections in Expt. 1.

\begin{tabular}{lcccc}
\hline & \multicolumn{4}{c}{ Salinity level $\left(\mathrm{dS} \cdot \mathrm{m}^{-1}\right)^{\mathrm{z}}$} \\
\cline { 2 - 5 } Selections & 12 & 24 & 36 & 48 \\
\hline CO-01 & $31.43 \mathrm{ef}^{\mathrm{y}}$ & $33.41 \mathrm{de}$ & $88.50 \mathrm{de}$ & $14.97 \mathrm{~h}$ \\
COAZ-19 & $34.66 \mathrm{def}$ & $92.33 \mathrm{ab}$ & $115.99 \mathrm{bcd}$ & $110.19 \mathrm{~b}$ \\
COAZ-15 & $28.14 \mathrm{ef}$ & $31.41 \mathrm{e}$ & $28.65 \mathrm{f}$ & $45.56 \mathrm{~g}$ \\
COAZ-16 & $55.46 \mathrm{cde}$ & $41.30 \mathrm{e}$ & $94.63 \mathrm{cde}$ & $69.00 \mathrm{de}$ \\
COAZ-20 & $72.09 \mathrm{bc}$ & $70.57 \mathrm{c}$ & $106.84 \mathrm{bcde}$ & $75.95 \mathrm{~cd}$ \\
COAZ-17 & $75.08 \mathrm{bc}$ & $118.72 \mathrm{a}$ & $103.09 \mathrm{bcde}$ & $65.90 \mathrm{def}$ \\
COAZ-06 & $24.70 \mathrm{f}$ & $52.50 \mathrm{c}$ & $72.69 \mathrm{e}$ & $51.61 \mathrm{efg}$ \\
COAZ-08 & $75.87 \mathrm{bc}$ & $69.10 \mathrm{~cd}$ & $123.94 \mathrm{bcd}$ & $76.04 \mathrm{~cd}$ \\
COAZ-11 & $87.87 \mathrm{~b}$ & $79.62 \mathrm{c}$ & $87.93 \mathrm{de}$ & $50.35 \mathrm{fg}$ \\
COAZ-21 & $60.43 \mathrm{bcd}$ & $91.35 \mathrm{~b}$ & $108.52 \mathrm{bcde}$ & $66.96 \mathrm{def}$ \\
COAZ-18 & $123.90 \mathrm{a}$ & $124.28 \mathrm{a}$ & $174.55 \mathrm{a}$ & $142.00 \mathrm{a}$ \\
COAZ-01 & $52.63 \mathrm{cdef}$ & $65.24 \mathrm{c}$ & $121.59 \mathrm{bcd}$ & $49.15 \mathrm{fg}$ \\
COAZ-22 & $55.00 \mathrm{cde}$ & $97.82 \mathrm{ab}$ & $136.20 \mathrm{~b}$ & $87.58 \mathrm{c}$ \\
COAZ-02 & $72.20 \mathrm{bc}$ & $78.77 \mathrm{c}$ & $129.79 \mathrm{bc}$ & $76.37 \mathrm{~cd}$ \\
LSD & 27.93 & 31.41 & 36.40 & 18.34
\end{tabular}

${ }^{\text {zThe }} 2 \mathrm{dS} \cdot \mathrm{m}^{-1}$ level was used as the covariate in statistical analysis, so it is not shown in the table.

${ }^{y}$ Means in the same column followed by the same letter are not significantly different at $P=0.05$.

Table 5. Leaf firing (0-100\%) of 12 saltgrass selections grown under different levels of salinity in Expt. 2.

\begin{tabular}{llllllll}
\hline & \multicolumn{4}{c}{ Salinity of culture solution $\left(\mathrm{dS} \cdot \mathrm{m}^{-1}\right)$} & & \\
Selections & \multicolumn{1}{c}{6} & \multicolumn{1}{c}{20} & \multicolumn{1}{c}{34} & \multicolumn{1}{c}{48} & & Linear regression & $R^{2}$ \\
\hline CO-01 & $37.75 \mathrm{ab}^{\mathrm{z}}$ & $30.21 \mathrm{~cd}$ & $45.21 \mathrm{ef}$ & $49.92 \mathrm{~cd}$ & & $Y=30.84+0.37 X$ & 0.25 \\
COAZ-23 & $40.71 \mathrm{ab}$ & $53.50 \mathrm{a}$ & $65.50 \mathrm{abcd}$ & $83.83 \mathrm{a}$ & $Y=33.62+1.01 X$ & $0.46^{*}$ \\
COAZ-24 & $39.42 \mathrm{ab}$ & $43.92 \mathrm{abc}$ & $66.71 \mathrm{abc}$ & $70.00 \mathrm{abc}$ & $Y=32.92+0.82 X$ & $0.39^{*}$ \\
COAZ-19 & $39.13 \mathrm{ab}$ & $33.67 \mathrm{bcd}$ & $47.46 \mathrm{def}$ & $41.25 \mathrm{~d}$ & & $Y=36.49+0.14 X$ & 0.07 \\
COAZ-16 & $47.46 \mathrm{a}$ & $49.67 \mathrm{ab}$ & $68.88 \mathrm{ab}$ & $72.21 \mathrm{ab}$ & $Y=41.53+0.67 X$ & $0.70^{* *}$ \\
COAZ-20 & $30.75 \mathrm{~b}$ & $38.04 \mathrm{abcd}$ & $50.08 \mathrm{cdef}$ & $51.08 \mathrm{~cd}$ & $Y=28.41+0.52 X$ & $0.69^{* *}$ \\
COAZ-17 & $31.75 \mathrm{~b}$ & $26.63 \mathrm{~d}$ & $39.92 \mathrm{f}$ & $67.83 \mathrm{abc}$ & $Y=18.09+0.87 X$ & $0.60^{* *}$ \\
COAZ-11 & $37.96 \mathrm{ab}$ & $40.33 \mathrm{abcd}$ & $54.50 \mathrm{bcdef}$ & $56.00 \mathrm{bcd}$ & $Y=34.03+0.49 X$ & 0.27 \\
COAZ-18 & $36.00 \mathrm{ab}$ & $36.79 \mathrm{bcd}$ & $52.83 \mathrm{bcdef}$ & $40.29 \mathrm{~d}$ & $Y=35.90+0.21 X$ & 0.12 \\
COAZ-01 & $31.04 \mathrm{~b}$ & $36.00 \mathrm{bcd}$ & $45.25 \mathrm{ef}$ & $51.17 \mathrm{~cd}$ & $Y=27.44+0.50 X$ & $0.63^{* *}$ \\
COAZ-22 & $37.88 \mathrm{ab}$ & $39.29 \mathrm{abcd}$ & $63.17 \mathrm{abcde}$ & $65.83 \mathrm{abc}$ & $Y=30.77+0.77 X$ & $0.68^{* *}$ \\
COAZ-02 & $44.17 \mathrm{a}$ & $45.92 \mathrm{abc}$ & $80.42 \mathrm{a}$ & $80.83 \mathrm{a}$ & $Y=34.97+1.03 X$ & $0.70^{* *}$ \\
LSD & 12.34 & 16.68 & 18.78 & 20.8 & & \\
\hline
\end{tabular}

${ }^{\mathrm{z}}$ Means in the same column followed by the same letter are not significantly different at $P=0.05$. $*, * *$ Significant linear relationship at $P=0.05$ or 0.01 , respectively.

and COAZ-01 consistently exhibited the highest turf quality throughout the 12-week period. All saltgrass selections exhibited lower turf quality rating than in Expt 1 . This was in part because some tillers died off even when they were grown under lowsalinity water and nutrient-unlimited envi- ronments. This may related to the fact that saltgrass was grown in space-limiting containers (cups) in our hydroponic systems. A similar observation was made in the field when saltgrass was grown in space-limiting boxes. We are unsure of the cause of this phenomenon. 
Table 6. Turf quality (scale of 0 to 9 , with $9=$ "best") of 12 saltgrass selections after 3 mo grown in hydroponic solution at 4 different levels of salinity in Expt. 2.

\begin{tabular}{|c|c|c|c|c|c|c|}
\hline \multirow[b]{2}{*}{ Selections } & \multicolumn{4}{|c|}{ Salinity of culture solution $\left(\mathrm{dS} \cdot \mathrm{m}^{-1}\right)$} & \multirow[b]{2}{*}{ Regression } & \multirow[b]{2}{*}{$R^{2}$} \\
\hline & 6 & 20 & 34 & 48 & & \\
\hline$\overline{\mathrm{CO}-01}$ & $4.37 \mathrm{abc}^{\mathrm{z}}$ & $4.40 \mathrm{ab}$ & $3.93 \mathrm{ab}$ & $3.63 \mathrm{a}$ & $Y=4.60-0.02 X$ & $0.43^{*}$ \\
\hline COAZ-23 & $3.93 \mathrm{~cd}$ & $3.53 \mathrm{~d}$ & $2.83 \mathrm{~cd}$ & $1.57 \mathrm{e}$ & $Y=4.47-0.06 X$ & $0.70 * *$ \\
\hline COAZ-24 & $3.63 \mathrm{~d}$ & $3.30 \mathrm{~d}$ & $2.80 \mathrm{~d}$ & $2.27 \mathrm{de}$ & $Y=3.89-0.03 X$ & $0.61 * *$ \\
\hline COAZ-19 & $4.33 \mathrm{bc}$ & $4.47 \mathrm{ab}$ & $3.87 \mathrm{ab}$ & $3.67 \mathrm{a}$ & $Y=4.58-0.02 X$ & $0.43^{*}$ \\
\hline COAZ-16 & $3.67 \mathrm{~d}$ & $3.63 \mathrm{~cd}$ & $2.77 \mathrm{~d}$ & $2.63 \mathrm{~cd}$ & $Y=3.94-0.03 X$ & $0.67 * *$ \\
\hline COAZ-20 & $4.83 \mathrm{ab}$ & $4.17 \mathrm{bc}$ & $3.77 \mathrm{ab}$ & $3.37 \mathrm{ab}$ & $Y=4.96-0.03 X$ & $0.80 * *$ \\
\hline COAZ-17 & $4.53 \mathrm{abc}$ & $4.90 \mathrm{a}$ & $4.30 \mathrm{a}$ & $2.87 \mathrm{bcd}$ & $Y=5.23-0.04 X$ & $0.56^{* *}$ \\
\hline COAZ-11 & $4.40 \mathrm{abc}$ & $4.37 \mathrm{ab}$ & $3.57 \mathrm{abc}$ & $3.23 \mathrm{abc}$ & $Y=4.72-0.03 X$ & $0.48 * *$ \\
\hline COAZ-18 & $4.60 \mathrm{ab}$ & $4.53 \mathrm{ab}$ & $3.27 \mathrm{bcd}$ & $3.90 \mathrm{a}$ & $Y=4.72-0.02 X$ & $0.38^{*}$ \\
\hline COAZ-01 & $4.97 \mathrm{a}$ & $4.57 \mathrm{ab}$ & $4.03 \mathrm{a}$ & $3.40 \mathrm{ab}$ & $Y=5.25-0.04 X$ & $0.79 * *$ \\
\hline COAZ-22 & $4.60 \mathrm{ab}$ & $4.53 \mathrm{ab}$ & $3.27 \mathrm{bcd}$ & $2.90 \mathrm{bcd}$ & $Y=5.05-0.05 X$ & $0.69 * *$ \\
\hline COAZ-02 & $4.23 \mathrm{bcd}$ & $3.57 \mathrm{~d}$ & $2.60 \mathrm{~d}$ & $2.23 \mathrm{de}$ & $Y=4.50-0.05 X$ & $0.85^{* *}$ \\
\hline LSD & 0.63 & 0.58 & 0.74 & 0.72 & & \\
\hline
\end{tabular}

${ }^{\mathrm{z}}$ Means in the same column followed by the same letter are not significantly different at $P=0.05$.

*,**Significant linear relationship at $P=0.05$ or 0.01 , respectively.

Table 7. Root mass (shown as grams per tube) of 12 saltgrass selections grown in hydroponic solution at 4 different levels of salinity in Expt. 2.

\begin{tabular}{llllllll}
\hline & \multicolumn{5}{c}{ Salinity of culture solution $\left(\mathrm{dS} \cdot \mathrm{m}^{-1}\right)$} & & \\
\cline { 2 - 5 } Selections & \multicolumn{1}{c}{6} & \multicolumn{1}{c}{20} & \multicolumn{1}{c}{34} & \multicolumn{1}{c}{48} & & Regression & $R^{2}$ \\
\hline CO-01 & $0.45 \mathrm{ab}^{2}$ & $0.68 \mathrm{ab}$ & $0.91 \mathrm{a}$ & $0.77 \mathrm{abc}$ & $Y=0.47+0.009 X$ & 0.18 \\
COAZ-23 & $0.18 \mathrm{~cd}$ & $0.27 \mathrm{~d}$ & $0.13 \mathrm{~d}$ & $0.03 \mathrm{~d}$ & $Y=0.27-0.004 X$ & 0.16 \\
COAZ-24 & $0.29 \mathrm{bcd}$ & $0.51 \mathrm{bcd}$ & $0.41 \mathrm{~d}$ & $0.20 \mathrm{~cd}$ & $Y=0.34+0.001 X$ & 0.01 \\
COAZ-19 & $0.40 \mathrm{abc}$ & $0.44 \mathrm{bcd}$ & $0.30 \mathrm{~cd}$ & $1.12 \mathrm{ab}$ & $Y=0.17+0.015 X$ & 0.29 \\
COAZ-16 & $0.10 \mathrm{~d}$ & $0.25 \mathrm{~d}$ & $0.22 \mathrm{~d}$ & $0.42 \mathrm{~cd}$ & $Y=0.06+0.007 X$ & $0.43^{*}$ \\
COAZ-20 & $0.49 \mathrm{ab}$ & $0.63 \mathrm{ab}$ & $0.99 \mathrm{a}$ & $1.22 \mathrm{a}$ & $Y=0.34+0.018 X$ & $0.56^{* *}$ \\
COAZ-17 & $0.30 \mathrm{bcd}$ & $0.61 \mathrm{abc}$ & $0.67 \mathrm{abc}$ & $0.47 \mathrm{bcd}$ & $Y=0.40+0.004 X$ & 0.08 \\
COAZ-11 & $0.34 \mathrm{abc}$ & $0.51 \mathrm{bcd}$ & $0.64 \mathrm{abc}$ & $1.16 \mathrm{a}$ & $Y=0.16+0.020 X$ & 0.33 \\
COAZ-18 & $0.18 \mathrm{~cd}$ & $0.40 \mathrm{bcd}$ & $0.45 \mathrm{bcd}$ & $1.09 \mathrm{ab}$ & $Y=-0.01+0.020 X$ & $0.65^{* *}$ \\
COAZ-01 & $0.17 \mathrm{~cd}$ & $0.50 \mathrm{bcd}$ & $0.71 \mathrm{ab}$ & $0.91 \mathrm{abc}$ & $Y=0.11+0.017 X$ & $0.71^{* *}$ \\
COAZ-22 & $0.53 \mathrm{a}$ & $0.88 \mathrm{a}$ & $0.47 \mathrm{bcd}$ & $0.90 \mathrm{abc}$ & $Y=0.56+0.005 X$ & 0.04 \\
COAZ-02 & $0.26 \mathrm{bcd}$ & $0.31 \mathrm{~cd}$ & $0.30 \mathrm{~cd}$ & $0.31 \mathrm{~cd}$ & $Y=0.27+0.001 X$ & 0.02 \\
LSD & 0.23 & 0.32 & 0.39 & 0.66 & & & \\
\hline
\end{tabular}

${ }^{\mathrm{z}}$ Means in the same column followed by the same letter are not significantly different at $P=0.05$.

**** Significant linear relationship at $P=0.05$ or 0.01 , respectively.

Table 8. Clipping yields (in grams per cup per week) of 12 saltgrass selections grown in hydroponic solution at 4 different levels of salinity in Expt. 2.

\begin{tabular}{llllll}
\hline & \multicolumn{4}{c}{ Salinity of culture solution $\left(\mathrm{dS} \cdot \mathrm{m}^{-1}\right)$} & \multicolumn{1}{c}{48} \\
\cline { 2 - 5 } Selections & \multicolumn{1}{c}{6} & \multicolumn{1}{c}{20} & \multicolumn{1}{c}{34} & $\mathrm{EC}_{25}\left(\mathrm{dS} \cdot \mathrm{m}^{-1}\right)^{\mathrm{y}}$ \\
\hline CO-01 & $0.42 \mathrm{bc}^{\mathrm{z}}$ & $0.47 \mathrm{ab}$ & $0.34 \mathrm{abc}$ & $0.25 \mathrm{abc}$ & 29.00 \\
COAZ-23 & $0.16 \mathrm{de}$ & $0.19 \mathrm{~cd}$ & $0.15 \mathrm{cde}$ & $0.07 \mathrm{ef}$ & 29.50 \\
COAZ-24 & $0.11 \mathrm{e}$ & $0.18 \mathrm{~cd}$ & $0.06 \mathrm{e}$ & $0.07 \mathrm{f}$ & 23.25 \\
COAZ-19 & $0.70 \mathrm{a}$ & $0.54 \mathrm{ab}$ & $0.42 \mathrm{ab}$ & $0.26 \mathrm{abc}$ & 22.00 \\
COAZ-16 & $0.17 \mathrm{de}$ & $0.20 \mathrm{~cd}$ & $0.08 \mathrm{de}$ & $0.07 \mathrm{def}$ & 21.17 \\
COAZ-20 & $0.53 \mathrm{abc}$ & $0.46 \mathrm{ab}$ & $0.34 \mathrm{abc}$ & $0.32 \mathrm{a}$ & 29.0 \\
COAZ-17 & $0.47 \mathrm{bc}$ & $0.37 \mathrm{bc}$ & $0.50 \mathrm{a}$ & $0.20 \mathrm{abcde}$ & 28.0 \\
COAZ-11 & $0.49 \mathrm{bc}$ & $0.48 \mathrm{ab}$ & $0.34 \mathrm{abc}$ & $0.21 \mathrm{abcd}$ & 24.86 \\
COAZ-18 & $0.34 \mathrm{~cd}$ & $0.37 \mathrm{abc}$ & $0.18 \mathrm{ced}$ & $0.26 \mathrm{ab}$ & 29.9 \\
COAZ-01 & $0.61 \mathrm{ab}$ & $0.56 \mathrm{a}$ & $0.34 \mathrm{abc}$ & $0.33 \mathrm{a}$ & 25.13 \\
COAZ-22 & $0.38 \mathrm{c}$ & $0.43 \mathrm{ab}$ & $0.29 \mathrm{bcd}$ & $0.14 \mathrm{bcdef}$ & 24.08 \\
COAZ-02 & $0.34 \mathrm{~cd}$ & $0.11 \mathrm{~d}$ & $0.21 \mathrm{bcde}$ & $0.13 \mathrm{cdef}$ & 23.25 \\
LSD & 0.21 & 0.19 & 0.21 & 0.14 & \\
\hline
\end{tabular}

${ }^{\mathrm{z}}$ Means in the same column followed by the same letter are not significantly different at $P=0.05$.

${ }^{\mathrm{y}}$ Salinity level (EC) resulting in $25 \%$ reduction in clipping yield.

Root production by the different saltgrass lines responded differently to increasing salinity (Table 7). Experimental lines COAZ-01, COAZ-18, COAZ-20, and COAZ-16 showed linear increases in root mass as salinity of the growth solution increased from 6 to $48 \mathrm{dS} \cdot \mathrm{m}^{-1}$, while COAZ-23 and COAZ-24 exhibited a decrease in root mass as salinity increased from 20 to $48 \mathrm{dS} \cdot \mathrm{m}^{-1}$. Root mass of CO-01 and COAZ-17 increased as salinity level increased from control $\left(6 \mathrm{dS} \cdot \mathrm{m}^{-1}\right)$ to 34 $\mathrm{dS} \cdot \mathrm{m}^{-1}$; with a further increase in salinity, root growth decreased. At the highest salinity level $\left(48 \mathrm{dS} \cdot \mathrm{m}^{-1}\right)$, experimental lines COAZ20, COAZ-19, COAZ-11, and COAZ-18 had higher root mass than COAZ-23, COAZ-24, COAZ-16, and COAZ-02. From Expt. 1 and 2 , we found that moderate levels of salinity increase saltgrass root biomass. Dudeck et al.
(1983) and Qian et al. (2000) also found that, as the salt concentration increased, root growth of bermudagrass and zoysiagrass increased to a maximum point and then declined. It is speculated that these plants require sodium or chloride for growth (Munns and Termatt, 1986).

Clipping yield is one indicator of turf vigor. All lines exhibited decreased clipping yield over time with increasing salinity (Table 8). During the 12-week exposure to $48 \mathrm{dS} \cdot \mathrm{m}^{-1}$, experimental lines COAZ-01, COAZ-20, COAZ-18, COAZ-19, and CO-01 exhibited higher clipping yields than did lines COAZ-23, COAZ-24, and COAZ-16. Regression analysis between clipping yield and growth medium salinity was used to derive $25 \%$ clipping yield reduction salinity level for each line. Salinity levels that caused a $25 \%$ clipping reduction ranged from $21.2 \mathrm{dS} \cdot \mathrm{m}^{-1}$ for COAZ-16 to $29.9 \mathrm{dS} \cdot \mathrm{m}^{-1}$ for COAZ-18, although the EC level that resulted in $25 \%$ clipping yield reduction was not different among experimental lines at $P<$ 0.05 (Table 8 ). In previous work, the $25 \%$ clipping yield reduction salinity has been used as an indicator of salinity tolerance (Fu et al., 2005; Qian et al., 2001, 2004; Suplick-Ploense et al., 2002). It was reported that the salinity (EC) of $25 \%$ clipping yield reduction ranged from 2.3 to $10.0 \mathrm{dS} \cdot \mathrm{m}^{-1}$ for Kentucky bluegrass (Qian et al., 2004; Suplick-Ploense et al., 2002) and from 5.7 to $14.0 \mathrm{dS} \cdot \mathrm{m}^{-1}$ for creeping bentgrass $(\mathrm{Fu}$ et al., 2005; Qian and Fu, 2005). Lee et al. (2005) reported that the salinity level resulting in $50 \%$ shoot growth reduction ranged from 28.8 to $39.3 \mathrm{dS} \cdot \mathrm{m}^{-1}$ for seashore paspalum (Paspalum vaginatum Swartz). The high EC value observed to result in $25 \%$ clipping yield reduction for saltgrass would rank it as one of the most salt-tolerant turfgrass species.

Based on the number of times in the best statistical category for turf quality, leaf firing, clipping yield, and root growth in Expt. 2, selections COAZ-18, COAZ-19, COAZ-20, and COAZ-01 demonstrated superior salinity tolerance, followed by CO-01 and COAZ-11.

In summary, our research suggests that saltgrass possesses excellent salt tolerance and that turf-type selections will potentially be among the most salt-tolerant turfgrasses. Our results also agree with those of Marcum et al. (2005) that a substantial range of salinity tolerance exists among saltgrass germplasms. Selections COAZ-18, COAZ-01, and CO-01 exhibited the best turf quality and good salt tolerance in both Expts. 1 and 2 . Salt excretion via salt glands, root growth stimulation under saline conditions, maintenance of high root-to-shoot ratio, regulation of ion concentrations, and maintenance of higher $\mathrm{K}^{+} / \mathrm{Na}^{+}$ratios in shoots are important salinity- tolerance mechanisms in saltgrass (Alshammary et al., 2004; Warren and Gould, 1982). Our experiment also demonstrated that, despite its halophytic behavior, saltgrass is not immune to salinity stress; it may suffer salinity stress as the level of salinity increases in the growth medium, as 
indicated by increased leaf firing and reduced turf quality and shoot growth.

The salinity screening procedures used in these experiments will be both valuable and efficient for screening parental germplasm in our breeding program. Further, turf-type lines developed by breeders can be tested for salinity tolerance before release or further development.

\section{Literature Cited}

Alshammary, S., Y.L. Qian, and S.J. Wallner. 2004. Growth response of four turfgrasses to salinity. Agr. Water Mgt. 66:97-111.

Aschenbach, T.A. 2006. Variation in growth rates under saline conditions of Pascoprum smithii (western wheatgrass) and Distichlis spicata (inland saltgrass) from different source populations in Kansas and Nebraska: implications for the restoration of salt-affected plant communities. Restor. Ecol. 14:21-27.

Carrow, R.N. and R.R. Duncan. 1998. Salt-affected turfgrass sites: assessment and management. Ann Arbor Press, Chelsea, Mich.

Dudeck, A.E., S. Singh, C.E. Giordano, T.A. Nell, and D.B. McConnell. 1983. Effects of sodium chloride on Cynodon turfgrasses. Agron. J. 75:927-930.

Fu, J.M., A.J. Koski, and Y.L. Qian. 2005. Responses of creeping bentgrass to salinity and mowing management: growth and turf quality. HortScience 40:463-467.

Gould, F.W. 1968. Grass systematics. McGrawHill Book Company, New York.

Hansen, D.J., P. Dayanandan, P.B. Kaufman, and J.D. Brotherson. 1976. Affecting its growth ecological adaptations of salt marsh grass, Distichlis spicata
(Gramineae), and environmental factors and distribution. Amer. J. Bot. 63:635-650.

Joslin, J.D. and G.S. Henderson. 1984. The determination of percentages of living tissue in woody fine root samples using triphenyltetrazolium chloride. Forest Sci. 30:965-970.

Kopec, D.M. and K.B. Marcum. 2001. Desert saltgrass: a potential new turfgrass species. U.S. Golf Assn. Green Sect. Rec. 39(1):6-8.

Lee, G.J., R.N. Carrow, and R.R. Duncan. 2005. Growth and water relation responses to salinity stress in halophytic seashore paspalum ecotypes. Sci. Hort. 104:221-236.

Marcum, K.B., M. Pessarakli, and D.M. Kopec. 2005. Relative salinity tolerance of 21 turf type desert saltgrass compared to bermudagrass. HortScience 40:227-229.

Munns, R. and A. Termatt. 1986. Whole-plant responses to salinity. Aust. J. Plant Physiol. 13:143-160.

Nielson, A.K. 1956. A study of the variability of Distichlis stricta selections from several geographical locations in the western United States. Utah State Agr. College, Logan, Utah, MS thesis. $46 \mathrm{pp}$.

O'Leary, J.W. and E.P. Glenn. 1994. Global distribution and potential for halophytes, $\mathrm{p}$. 7-19. In: V.R. Squires A.T. Ayoub (eds.). Halophytes as a resource for livestock and for rehabilitation of degraded lands. Kluwer Acad. Publ., Dordrecht.

Qian, Y.L., M.C. Engelke, and M.J.V. Foster. 2000. Salinity effects on zoysiagrass cultivars and experimental lines. Crop Sci. 40:488-492.

Qian, Y.L., R.F. Follett, S. Wilhelm, A.J. Koski, and M.A. Shahba. 2004. Carbon isotope discrimination of three Kentucky bluegrass cultivars with contrasting salinity tolerance. Agron. J. 96:571-575.
Qian, Y.L. and J.M. Fu. 2005. Response of creeping bentgrass to salinity and mowing management: carbohydrate availability and ion accumulation. HortScience 40:2170-2174.

Qian, Y.L., A.J. Koski, and R. Welton. 2001. Amending isolite and zeolite in sand under saline conditions: leachate composition and salt deposition. HortScience 36:717-720.

Qian, Y.L. and B. Mecham. 2005. Long-term effects of recycled wastewater irrigation on soil chemical properties on golf course fairways. Agron. J. 97:717-721.

Qian, Y.L., S.J. Wilhelm, and K.B. Marcum. 2001. Comparative responses of two Kentucky bluegrass cultivars to salinity stress. Crop Sci. 41:1895-1900.

SAS Institute. 2006. SAS/STAT user's guide. SAS Institute, Cary, N.C.

Shahba, M.A., Y.L. Qian, H.G. Hughes, D. Christensen, and A.J. Koski. 2003a. Cold hardiness of saltgrass accessions. Crop Sci. 43:21422147.

Shahba, M.A., Y.L. Qian, H.G. Hughes, A.J Koski, and D. Christensen. 2003b. Relationship of carbohydrates and cold hardiness in six saltgrass accessions. Crop Sci. 43:2148-2153.

Suplick-Ploense, M.R., Y.L. Qian, and J.C. Read 2002. Salinity tolerance of Texas bluegrass, Kentucky bluegrass, and their hybrids. Crop Sci. 42:2025-2030.

Ungar, I.A. 1974. Inland halophytes of the United States, p. 235-305. In: R. Reimold and E. Queen (eds.). Ecology of halophytes. Academic Press, New York.

Warren, R.S. and A.R. Gould. 1982. Salt tolerance expressed as a cellular trait in suspension cultures developed from the halophytic grass Distichlis spicata. Z. Pflanzenphysiol. Bd. 107:347-356. 